\title{
Transgenerational impact of intimate partner violence on methylation in the promoter of the glucocorticoid receptor
}

\author{
KM Radtke ${ }^{1,2,4}$, M Ruf $^{1,4}$, HM Gunter ${ }^{2,3,4}$, K Dohrmann $^{1}$, M Schauer ${ }^{1}$, A Meyer ${ }^{2}$ and T Elbert ${ }^{1}$
}

Prenatal exposure to maternal stress can have lifelong implications for psychological function, such as behavioral problems and even the development of mental illness. Previous research suggests that this is due to transgenerational epigenetic programming of genes operating in the hypothalamic-pituitary-adrenal axis, such as the glucocorticoid receptor (GR). However, it is not known whether intrauterine exposure to maternal stress affects the epigenetic state of these genes beyond infancy. Here, we analyze the methylation status of the GR gene in mothers and their children, at 10-19 years after birth. We combine these data with a retrospective evaluation of maternal exposure to intimate partner violence (IPV). Methylation of the mother's GR gene was not affected by IPV. For the first time, we show that methylation status of the GR gene of adolescent children is influenced by their mother's experience of IPV during pregnancy. As these sustained epigenetic modifications are established in utero, we consider this to be a plausible mechanism by which prenatal stress may program adult psychosocial function.

Translational Psychiatry (2011) 1, e21; doi:10.1038/tp.2011.21; published online 19 July 2011

\section{Introduction}

Prenatal stress can have a lasting detrimental impact on psychological health; however, the molecular mechanisms that transmit this experience to adult behavior are not fully characterized. $^{1-5}$ The hypothalamic-pituitary-adrenal (HPA) axis is critical for homeostasis-it controls growth, reproduction, metabolism and behavior; it is also the primary line of the 'defence cascade' that helps humans to deal with crises. Hyperactivity of the HPA-axis can cause anything from a longlasting head cold to depression. Hypoactivity of the HPA-axis can cause undesirable consequences such as abdominal fat, loss of muscle mass and mental ill-health.,7 Tuning of the HPA-axis and its physiological pathways is highly susceptible to the influence of early life events. Prenatal stress such as antenatal exposure to maternal anxiety has sustained effects on HPA-axis function ${ }^{8}$ and is associated with behavioral and emotional problems arising during development. ${ }^{9,10}$

DNA methylation could be a mechanism by which prenatal stress is translated into changes in gene expression and physiology, ${ }^{11}$ and ultimately psychologically vulnerable phenotypes. ${ }^{12,13}$ The glucocorticoid receptor (GR), a major regulator of the HPA-axis, ${ }^{14}$ could be involved in such a transmission, controlling many aspects of development, metabolism and immune function. Landmark experiments in rodents show that both the hippocampal expression of this gene and the behavioral responses to stress are modulated by the amount of care mothers invest into their offspring in the first days of postnatal life. ${ }^{15,16}$ This is likely to be the result of epigenetic modifications, specifically, through methylation of exon $1 \mathrm{~F}$ of the $G R$ promoter. ${ }^{17}$ This exon contains a response element for the nerve-growth-factor-inducible protein $A$ (NGFI-A), ${ }^{18}$ and binding of NGFI-A to its response element increases $G R$ expression. Methylation of the NGFI-A response element inhibits the association with its 'ligand' thereby decreasing $G R$ expression. ${ }^{19,20}$ Also, the human $G R$ gene is affected by aversive social environments, as childhood abuse leads to increased methylation in exon $1 \mathrm{~F}$ of the GR promoter. ${ }^{21}$

The vast majority of studies analyzing the influence of stress on the epigenetic regulation of the HPA-axis have focussed on postnatal stressors. However, recent research suggests that the intrauterine environment can also impact the epigenetic state of HPA-axis genes-prenatal exposure to maternal depressive mood was shown to correlate with $G R$ promoter methylation in newborns. ${ }^{22}$ Furthermore, increased GR promoter methylation was associated with higher cortisol responses to stress in this study and could therefore represent fetal programming of the HPA-axis. However, this study focused on methylation status in umbilical cord blood. Therefore, it is not known whether epigenetic marks that are established in utero due to the psychosocial situation of the mother are maintained beyond infancy.

Here, we investigate whether gestational maternal aversive experiences can have a prolonged effect, 10-19 years after birth, on DNA methylation of the offspring based on bisulfite sequencing of DNA from whole blood. Both intrauterine exposure to marital discord and $G R$ promoter methylation

\footnotetext{
${ }^{1}$ Department of Psychology, University of Konstanz and Center for Psychiatry Reichenau, Konstanz, Germany; ${ }^{2}$ Lehrstuhl für Zoologie und Evolutionsbiologie, Department of Biology, University of Konstanz, Konstanz, Germany and ${ }^{3}$ Zukunftskolleg, University of Konstanz, Konstanz, Germany

Correspondence: Professor T Elbert, Department of Psychology, University of Konstanz and Center for Psychiatry Reichenau, 78457 Konstanz, Germany or Professor A Meyer, Lehrstuhl für Zoologie und Evolutionsbiologie, Department of Biology, University of Konstanz, 78457 Konstanz, Germany.

E-mail: thomas.elbert@uni-konstanz.de or axel.meyer@uni-konstanz.de

${ }^{4}$ These authors contributed equally to this work.

Keywords: epigenetics; glucocorticoid receptor; hypothalamic-pituitary-adrenal axis; intimate partner violence; methylation; prenatal stress

Received 6 June 2011; accepted 7 June 2011
} 
can predict psychopathology. ${ }^{21,23,24}$ We aimed to determine whether prenatal exposure to intimate partner violence (IPV) leads to increased $G R$ promoter methylation later in life, which might mediate increased susceptibility to psychopathology.

\section{Materials and methods}

Participants. The study cohort represents a convenience sample, as there were no specific criteria that the participants had to fulfil. Mother-child pairs were either recruited via advertisements or taken from another study. ${ }^{25}$

Psychological parameters. We studied the impact of exposure to IPV in women from a variety of ethnic backgrounds (for example, Kosovo, Russia, Turkey etc.), who reside in Germany (Table 1). IPV was evaluated using the composite abuse scale (CAS) ${ }^{26}$ applied as structured interview by experienced clinical psychologists. The CAS is a validated tool, ${ }^{27}$ which measures the degree of domestic violence experienced by an individual in the four dimensions of severe combined abuse, physical abuse, emotional abuse and harassment. ${ }^{26}$ It consists of 30 items, which are scaled from 0 (never) to 5 (daily). Missing items, due to problems concerning the translation, were replaced by the mean of the existing items ( 1 of 25 interviews). According to previously described methods, ${ }^{28,29}$ a resulting 'sumscore' of 7 or higher was used as the criterion for exposure to IPV. In order to match the design of our study, the CAS was conducted three times separately focusing on the periods before, during and after the pregnancy with the particular child whose blood was analyzed in this study. For the periods before and after pregnancy, participants were asked to report acts of domestic violence whenever these happened before or after pregnancy with the relevant child. These periods were not limited to an absolute time span.

Sodium bisulfite sequencing. Sodium bisulfite conversion was performed, according to previously described methods, ${ }^{30,31}$ on DNA extracted from whole blood (DNeasy Blood and Tissue Kit, Qiagen, Hilden, Germany). Briefly, $5 \mu \mathrm{g}$ of DNA was denatured by incubating in $0.4 \mathrm{M} \mathrm{NaOH}$ (Riedel-deHaën, Seelze, Germany) for $30 \mathrm{~min}$ at $42^{\circ} \mathrm{C}$. After the addition of hydroquinone (Sigma-Aldrich, Steinheim, Germany) and sodium bisulfite (Sigma-Aldrich) to a final concentration of $0.5 \mathrm{~mm}$ and $2.6 \mathrm{~mm}$, respectively, the reaction mixture was incubated at $55^{\circ} \mathrm{C}$ for $18 \mathrm{~h}$. To remove free sodium bisulfite, the reaction mixture was cleaned up using a silica-based method, ${ }^{32}$ and was eluted in $100 \mu \mathrm{l}$ of double-distilled $\mathrm{H}_{2} \mathrm{O}$. To remove the bisulfite adduct, $11 \mu \mathrm{l}$ of $4.0 \mathrm{M} \mathrm{NaOH}$ was added, and the reaction mixture was incubated at $37^{\circ} \mathrm{C}$ for $15 \mathrm{~min}$. The reaction mixture was then neutralized by adding $111 \mu \mathrm{l}$ of $7.5 \mathrm{M}$ ammonium acetate, $\mathrm{pH}$ 7.0, (Riedel-deHaën). DNA was precipitated with ethanol $^{33}$ and resuspended in $30 \mu \mathrm{l}$ of double-distilled $\mathrm{H}_{2} \mathrm{O}$.

Table 1 Maternal IPV and sociodemographics

\begin{tabular}{|c|c|c|c|c|c|c|c|c|c|}
\hline \multirow[t]{2}{*}{ Label } & \multicolumn{4}{|c|}{ Maternal exposure to IPV } & \multirow{2}{*}{$\begin{array}{c}\text { Children's } \\
\text { gender }\end{array}$} & \multirow{2}{*}{$\begin{array}{c}\text { Children's } \\
\text { age } \\
\text { (years) }\end{array}$} & \multirow[t]{2}{*}{ Mode of birth } & \multirow{2}{*}{$\begin{array}{c}\text { Maternal } \\
\text { age at } \\
\text { birth (years) }\end{array}$} & \multirow{2}{*}{$\begin{array}{l}\text { Maternal } \\
\text { country } \\
\text { of origin }\end{array}$} \\
\hline & $\begin{array}{l}\text { Before } \\
\text { pregnancy }\end{array}$ & $\begin{array}{l}\text { During } \\
\text { pregnancy }\end{array}$ & $\begin{array}{l}\text { After } \\
\text { pregnancy }\end{array}$ & Total & & & & & \\
\hline 1 & No & No & No & No & Male & 19 & Spontaneous birth & 19 & Rus. \\
\hline 2 & No & No & No & No & Male & 17 & Spontaneous birth & 31 & PI. \\
\hline 3 & No & No & No & No & Female & 13 & Emergency caesarean section & 25 & $\mathrm{Tr}$ \\
\hline 4 & No & No & No & No & Male & 10 & Spontaneous birth & 29 & Ger. \\
\hline 5 & No & No & Yes & Yes & Female & 16 & Spontaneous birth & 19 & Irq. \\
\hline 6 & No & No & No & No & Female & 14 & Elective caesarean delivery & 28 & Ger. \\
\hline 7 & No & No & No & No & Male & 13 & Spontaneous birth & 28 & Rus. \\
\hline 8 & No & No & No & No & Female & 14 & Elective caesarean delivery & 27 & Rus. \\
\hline 10 & No & Yes & Yes & Yes & Female & 15 & Other & 29 & Ger. \\
\hline 11 & No & No & Yes & Yes & Female & 12 & Spontaneous birth & 31 & Tw. \\
\hline 12 & No & No & Yes & Yes & Male & 17 & Emergency caesarean section & 21 & Ir. \\
\hline 13 & No & No & No & No & Female & 17 & Spontaneous birth & 26 & Cs. \\
\hline 14 & No & Yes & No & Yes & Female & 13 & Spontaneous birth & 38 & Rus. \\
\hline 17 & No data & No data & Yes & Yes & Female & 12 & Emergency caesarean section & 18 & Irq \\
\hline 18 & No & No & No & No & Female & 13 & Spontaneous birth & 29 & Sos. \\
\hline 20 & No & No & No & No & Female & 12 & Spontaneous birth & 26 & Sos. \\
\hline 21 & Yes & Yes & Yes & Yes & Male & 11 & Elective caesarean delivery & 23 & Sos. \\
\hline 22 & No & No & No & No & Male & 12 & Spontaneous birth & 20 & Scg. \\
\hline 23 & No & Yes & No & Yes & Female & 10 & Spontaneous birth & 19 & Irq. \\
\hline 24 & No & No & No & No & Male & 13 & Spontaneous birth & 29 & Sos. \\
\hline 25 & Yes & Yes & Yes & Yes & Female & 14 & Spontaneous birth & 24 & Sos. \\
\hline 26 & No & No & Yes & Yes & Female & 17 & Spontaneous birth & 16 & Sos. \\
\hline 27 & No data & Yes & No data & Yes & Female & 14 & Spontaneous birth & 22 & Tr. \\
\hline 28 & Yes & Yes & Yes & Yes & Female & 18 & Spontaneous birth & 22 & Tr. \\
\hline 29 & No data & Yes & No data & Yes & Female & 17 & Spontaneous birth & 30 & Tr. \\
\hline $\begin{array}{l}n \\
\text { Range } \\
\text { Mean } \pm \text { s.e.m. }\end{array}$ & $3(22)$ & $8(24)$ & $9(23)$ & $13(25)$ & 25 & $\begin{array}{c}25 \\
10-19 \\
14.1 \pm 0.5\end{array}$ & 25 & $\begin{array}{c}25 \\
16-38 \\
25.2 \pm 1.0\end{array}$ & 25 \\
\hline
\end{tabular}

Abbreviations: Cs., Czechoslovakia; Ger., Germany; IPV, intimate partner violence; Ir., Iran; Irq., Iraq; PI., Poland; Rus., Russia; Scg., Serbia and Montenegro; Sos., Kosovo; Tw., Taiwan; Tr., Turkey.

'Total' indicates exposure to IPV regardless of timing of exposure. Whenever IPV was experienced before, during or after pregnancy 'total' was computed as 'yes'. In three cases we could not obtain CAS-scores across all periods as indicated by 'no data'. 
The converted DNA was amplified with PCR, using FastStart Taq DNA Polymerase (Roche, Mannheim, Germany), with the previously published PCR-primers: ${ }^{34}$ forward, -142763905-5'-GTTGTTATTYGTAGGGGTATTGG-3'-1427 63883 and reverse, 142763770-5'-AAACCACCRAATTTCT CCAA-3'-142763789 (sequence numbering is according to the published nucleotide position on chromosome 5; GenBank accession number: AJ877168). PCR conditions: $94^{\circ} \mathrm{C}$ for $5 \mathrm{~min}$, then 34 cycles of denaturation $\left(30 \mathrm{~s}, 95^{\circ} \mathrm{C}\right)$, annealing $\left(2 \mathrm{~min}, 56^{\circ} \mathrm{C}\right)$ and elongation $\left(30 \mathrm{~s}, 72^{\circ} \mathrm{C}\right)$, plus a final elongation step $\left(72^{\circ} \mathrm{C}, 7 \mathrm{~min}\right)$. The PCR was designed to amplify a $93 \mathrm{bp}$ fragment in the GRpromoter spanning $10 \mathrm{CpG}$ sites. Products were purified (QIAquick, Qiagen) and then cloned into pCR4-TOPO-vector using the TOPO TA Cloning kit (Invitrogen, Darmstadt, Germany). Positive clones were identified with colony PCR using the primers designed to the plasmid: M13-Fwd and M13-Rev primers (Invitrogen). Between 14 and 25 clones were sequenced using the same forward and reverse primers used for colony PCR (BigDye Terminator 3.1 Cycle Sequencing kit, Applied Biosystems, Darmstadt, Germany). The sequences for each individual clone were aligned and analyzed in Sequencher (version 4.2.2., Gene Codes Corporation, Ann Arbour, MI, USA).

Statistical analysis. Statistical analysis was conducted using PASW statistics (version 18.0, SPSS incorporation, Munich, Germany). To test for a potential association between maternal exposure to IPV and the presence of methylation in either the mothers or the children, we conducted Fisher's exact tests thereby treating methylation as a binary variable. The $G R$ promoter was referred to as being methylated, if a methylated CpG site was detected in at least one of up to 25 clones that were sequenced. For the mothers, we did not distinguish between different periods of exposure to IPV. Therefore, we computed an overall variable for exposure to IPV, assigning exposure to IPV whenever it happened before, during or after pregnancy. For the children, as we were interested in the relationship between methylation and maternal exposure to IPV before, during and after pregnancy, we performed tests independently for each interval.

To test for an association between the methylation status of the GR promoter in the mothers and in the children, we used Fisher's exact test.

To further analyze the direction of the relationship between methylation and maternal exposure to IPV during pregnancy, we conducted a Man-Whitney U-test with the exposure to IPV during pregnancy as the between group factor, and the percentage of methylated clones in the children as the dependent variable. The percentage of methylated clones was calculated, according to previously described methods, ${ }^{21}$ as the number of clones containing at least one methylated $\mathrm{CpG}$ site divided by the total number of clones. Additionally, we analyzed the relationship between maternal exposure to IPV before and after gestation and the percentage of methylated clones in the children in the same way.

\section{Results}

Maternal stress profiling. A total of 25 women of various ethnic backgrounds (Table 1) participated in the study. They were aged between 29 and 51 years. All of them had children aged between 10 and 19 years (Table 1 ). We used the CAS to screen the mothers, but not the children, for exposure to IPV before, during and after their pregnancies. We identified three women, who were exposed to IPV in the period before pregnancy, eight women, who were exposed during and nine after pregnancy. It should be noted that these groups were overlapping, that is, for some women exposure to IPV was apparent in more than one period. In three cases we could not obtain CAS-values for each period, therefore, sample sizes varied slightly in the statistical analyses (Table 1).

$\boldsymbol{G R}$ promoter methylation. We examined the methylation status of $10 \mathrm{CpG}$ sites in the GR promoter, to determine whether there is a link between exposure to IPV and methylation in a gene that is involved in the HPA-axis. This region was selected because it was previously shown to contain transcription factor binding sites, whose methylation statuses are influenced by early life stress. ${ }^{21}$ The GR promoter was referred to as being methylated, if a methylated CpG site was detected in at least one of up to 25 clones that were sequenced. We characterized $G R$ promoter methylation of 25 women and of 24 of their children (for one mother, we could not obtain a blood sample of any of her children). We detected methylation in 7 of $10 \mathrm{CpG}$ sites (sites 01, 02, 03, 07, 08, 09 and 10) in the GR promoter in our samples. The degree of methylation in these CpG sites ranged from 0 to $20 \%$ of all analyzed clones (Figure 1).

We detected methylation in the GR promoter of 10 mothers and seven children in at least one clone. There was no association between maternal methylation status and the methylation status of the children $(n=24 ; P=1.0)$.

Impact of IPV on maternal methylation pattern. Using fisher's exact test, we did not find a significant relationship between exposure to IPV and maternal methylation status ( $n=25, P=0.7$ ), irrespective of when IPV was experienced.

Impact of IPV on methylation patterns in offspring. The presence of methylated residues was significantly associated with maternal exposure to IPV during pregnancy $(n=23$, $P<0.05)$. Remarkably, there was no association between maternal exposure to IPV and the presence of methylation in the offspring, when IPV was experienced either before or after pregnancy (before: $n=21, P=1.0$; after: $n=22$, $P=1.0)$. Furthermore, we tested the influence of a variety of third variables (country of origin; maternal age; age of children; marital status; graduation from high school, completion of vocational training or academic degree; pregnancy problems; smoking, alcohol or drug consumption during pregnancy; use of painkillers during birth; anxiety, panic or helplessness during birth; skin contact after birth; severe diseases of children; birth weight; gestational age at birth; mode of birth) on the methylation status, but did not find a significant relationship (Supplementary Table S1 and S2). According to the guidelines of the world health organization (WHO) none of the analyzed children were of extreme immaturity ( $<28$ completed weeks of gestation, WHO) or extreme low birth weight (birth weight of $999 \mathrm{~g}$ or less, WHO). 


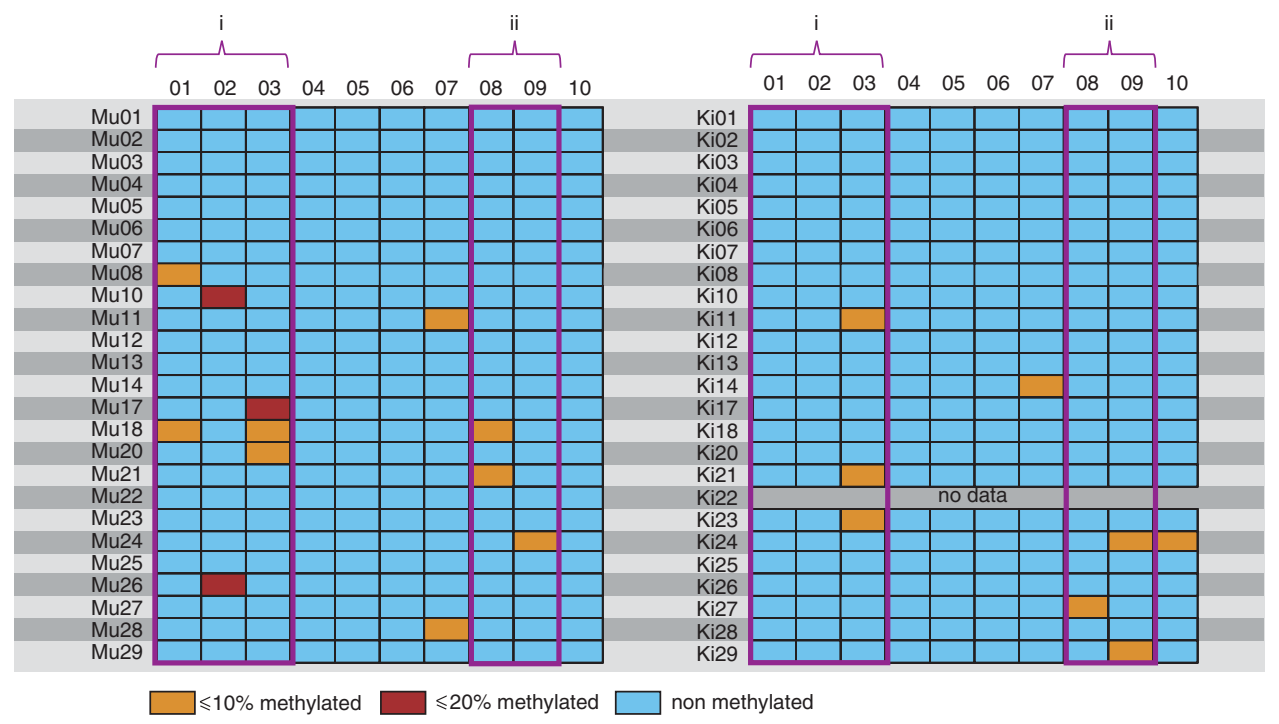

Figure 1 Degree of methylation per individual and $\mathrm{CpG}$ site. Each line represents the analyzed fraction of the glucocorticoid receptor gene of a woman (Mu) and her child (Ki). The rows correspond to individual $\mathrm{CpG}$ sites. The degree of methylation for each individual $\mathrm{CpG}$ site, that is, the number of clones containing methylation in the particular site divided by the total number of sequenced clones, is colour-coded. In the case of one child we could not obtain a blood sample as indicated by 'no data'. (i) Putative NGFI-Abinding site; (ii) Known NGFI-A-binding site.

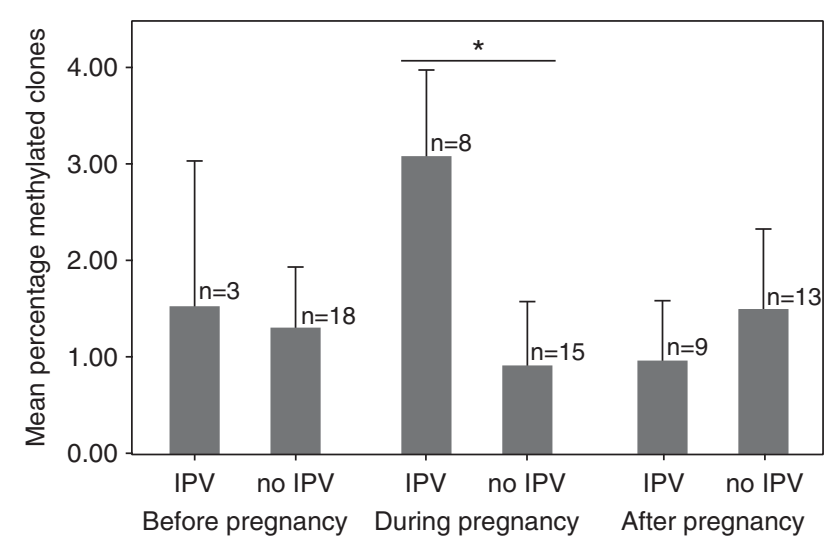

Figure 2 Gestational effects of IPV on methylation of the GR promoter in the children. Mean \pm s.e.m. of percentage of methylated clones for the children of women exposed to IPV. IPV only associates with increased methylation, if maternal exposure occurred during pregnancy. The percentage of methylated clones was calculated as the number of clones containing at least one methylated $\mathrm{CpG}$ site divided by the total number of clones. ${ }^{*} P<0.05$; IPV, intimate partner violence.

However, one child was born prematurely (28 completed weeks or more but less than 37 completed weeks of gestation, $\mathrm{WHO}$ ) and four children were born with low birth weights (1000-2499 g, WHO). None of these children showed methylation at the GR promoter. Furthermore, the vast majority of the children $(n=17)$ were delivered spontaneously, indicating that most mothers did not take any measures to delay or induce labor. However, six were delivered via caesarean section and one via a different procedure (Supplementary Table S2). Neither the birth weight, the gestational age nor the mode of birth affected the methylation status of the $G R$ gene (Supplementary Table S1). This provides evidence that methylation in the offspring is directly affected by adverse experiences of the mother during gestation.

We then sought to determine whether gestational exposure to IPV influenced the degree of methylation in the $G R$ promoter in the offspring. To achieve this we evaluated the percentage of methylated clones (that is, the number of clones containing at least one methylated CpG site divided by the total number of clones). This value ranged from 0 to $20 \%$ across all of our experimental subjects. In line with our previous analysis, we found a significant relationship between the percentage of methylated clones and IPV during pregnancy $(n=23, U=30.5, P=0.015$; Figure 2; Supplementary Figure S3). Additionally, we did not find an association between the percentage of methylated residues and maternal exposure to IPV before or after pregnancy (before: $n=21, U=25.0, P=0.9$; after: $n=22, U=55.0$, $P=0.7$; Figure 2; Supplementary Figure S4-5).

\section{Discussion}

We examined the methylation pattern of the $G R$ promoter in 25 women and their children and analyzed its association with maternal stress during gestation. We find a positive relationship between methylation of the children's GR promoter and maternal exposure to IPV during pregnancy, defined as 'any behavior within an intimate relationship that causes physical, psychological or sexual harm to those in the relationship. ${ }^{35}$ We present evidence that GR methylation is related to maternal stress during pregnancy, as when IPV is experienced before or after pregnancy, it has no impact on the children's methylation. To the best of our knowledge, this is the first study to demonstrate that prenatal psychological stress can result in sustained, rather than just short-term alteration of methylation in a regulator gene of the HPA-axis. 
We found that maternal gestational IPV is associated with methylation of exon $1 F$ in the GR promoter of the offspring, which is hence a transgenerational effect that may exert a lifelong influence on HPA-axis regulation in these individuals. This is consistent with previous observations that prenatal anxiety is associated with a sustained elevation of basal HPA activity $^{8}$ as well as with behavioral/emotional problems that may continue throughout the lifetime..$^{9,36}$ It seems likely that the intrauterine environment that the fetus experiences can differentially affect the methylation pattern. Maternal stress may lead to changes in HPA regulatory circuits with an alteration of catecholamines and glucocorticoids among other factors and effects. ${ }^{14}$ Prenatal exposure to either of these hormones is known to influence the development of the HPAaxis. $^{37}$ Therefore in the fetus, the GR gene, which is a key regulator of the HPA-axis, constitutes a likely target upon which maternal IPV could be acting. Our data are consistent with observations in rat, where maternal effects evoke increased HPA-axis activity and fearfulness in response to stress, which are associated with methylation of the GR promoter in the hippocampus. ${ }^{17}$ In these experiments, the first days of postnatal life constitute a critical period for the manifestation of maternal effects, which corresponds to late gestation in humans. ${ }^{38}$ Although the observed increase in methylation was only very subtle, it might be sufficent to alter HPA-axis function. Subtle increases in methylation in a single CpG site of the GR promoter in cord blood were reported to correlate positively with the cortsiol stress response in neonates. ${ }^{22}$ This suggests that IPV elicits fetal programming of the HPA-axis through methylation of the $G R$ gene. However, although a link between $G R$ promoter methylation in blood and cortisol stress responses has already been provided, ${ }^{22}$ it remains inconclusive whether methylation in blood cells reflects methylation in the hippocampus or whether it impacts HPA-axis function. This emphasizes the need for follow-up studies, which evaluate both HPA-axis function and psychological function. This is of particular interest, as blood-as opposed to brain tissue-represents an easily sampled tissue.

The results of our study suggest that maternal stressinduced changes in intrauterine environment can have an impact on the methylation pattern of the children's GR gene, rather than a direct maternal transmission of methylation pattern via the germ line. The CAS assesses exclusively environmental factors, episodes of domestic violence in this case, thereby minimizing the influence of genetic components. However, an individual's genotype might affect others' behavior towards them, thus their own social environment, or predispose them to seek risky or unfavorable social environments. ${ }^{39}$ As this type of indirect effect is difficult to avoid in studies with humans, we believe that our chosen methods go a long way to minimizing the influence of underlying genetic background. Hence, we consider gestational IPV to be the main source of variation in our study.

The methodologies employed by our study present some limitations: we have adapted a commonly used psychological survey - the CAS, to examine the influence of past events on current methylation patterns of mothers and their children. The effectiveness of retrospective surveys relies on the accuracy of our participants' memories. We believe that our use of an event-based analysis tool is particularly robust because of its reliance on emotionally arousing events, which are known to create long-lasting memories. ${ }^{40}$ Our data represent correlative findings and thus cannot prove a causal relationship between changes in methylation and adverse experiences. For instance, it might be possible that IPV correlates with aspects of maternal diet that in turn might have affected the observed methylation patterns. Such a scenario, however, seems unlikely, as we would then predict an association between IPV and methylation in the mothers. This, however, was not observed.

\section{Conclusions}

Our findings show that prenatal exposure to IPV is associated with a sustained increase in methylation of the human $G R$ promoter in the blood. Prenatal stress is known to alter HPAaxis regulatory function later in life. ${ }^{8}$ Specifically gestational marital discord is associated with psychopathology of the offspring. ${ }^{23,24}$ This is the first demonstration that gestational exposure to psychological stressors can have a lasting impact on methylation status in human offspring. Our results provide a potential mechanism-methylation of the GR promoterupon which prenatal stress could act, to influence psychological function. This emphasizes the importance of IPV interventions to assure the well-being not only of the mother but also of the unborn child. This mechanism opens up many new avenues for research on the transgenerational epigenetic effects of stress and aggression on human behavior.

\section{Conflict of interest}

The authors declare no conflict of interest.

Acknowledgements. We thank IT Kolassa, A Ashe, Ö Hatirnaz and WA Schulz for helpful suggestions on the methods, M Roth and $H$ Riedke for help during sample collection, and PM Hull and F Henning for helpful suggestions on the manuscript. HMG is supported by the Zukunftskolleg of University of Konstanz. Research was supported by the DFG.

1. St Clair $D, X u M$, Wang $P, Y u Y$, Fang $Y$, Zhang $F$ et al. Rates of adult schizophrenia following prenatal exposure to the Chinese famine of 1959-1961. JAMA 2005; 294: 557-562.

2. Susser E, Neugebauer R, Hoek HW, Brown AS, Lin S, Labovitz D et al. Schizophrenia after prenatal famine. Further evidence. Arch Gen Psychiatry 1996; 53: 25-31.

3. Susser ES, Lin SP. Schizophrenia after prenatal exposure to the Dutch Hunger Winter of 1944-1945. Arch Gen Psychiatry 1992; 49: 983-988.

4. Watson JB, Mednick SA, Huttunen M, Wang X. Prenatal teratogens and the development of adult mental illness. Dev Psychopathol 1999; 11: 457-466.

5. Talge NM, Neal C, Glover V. Antenatal maternal stress and long-term effects on child neurodevelopment: how and why? J Child Psychol Psychiatry 2007; 48: 245-261.

6. Elbert T, Rockstroh B. Stress factors. The science of our flexible responses to an unpredictable world. Nature 2003; 421: 477-478

7. McEwen BS, Lasley EN. The End of Stress as We know It. Joseph Henry Press/ Dana Press: Washington DC, USA, 2002.

8. O'Connor TG, Ben-Shlomo Y, Heron J, Golding J, Adams D, Glover V. Prenatal anxiety predicts individual differences in cortisol in pre-adolescent children. Biol Psychiatry 2005; 58: 211-217.

9. O'Connor TG, Heron J, Golding J, Beveridge M, Glover V. Maternal antenatal anxiety and children's behavioural/emotional problems at 4 years. Report from the Avon Longitudinal Study of Parents and Children. Br J Psychiatry 2002; 180: 502-508.

10. O'Connor TG, Heron J, Golding J, Glover V. Maternal antenatal anxiety and behavioural/ emotional problems in children: a test of a programming hypothesis. J Child Psychol Psychiatry 2003; 44: 1025-1036. 
11. Murgatroyd C, Patchev AV, Wu Y, Micale V, Bockmuhl Y, Fischer D et al. Dynamic DNA methylation programs persistent adverse effects of early-life stress. Nat Neurosci 2009; 12 : 1559-1566.

12. Gregory SG, Connelly JJ, Towers AJ, Johnson J, Biscocho D, Markunas CA et al. Genomic and epigenetic evidence for oxytocin receptor deficiency in autism. BMC Med 2009; 7: 62.

13. Uddin M, Aiello AE, Wildman DE, Koenen KC, Pawelec G, de Los Santos R et al. Epigenetic and immune function profiles associated with posttraumatic stress disorder. Proc Natl Acad Sci USA 2010; 107: 9470-9475.

14. de Kloet ER, Joels M, Holsboer F. Stress and the brain: from adaptation to disease. Nat Rev Neurosci 2005; 6: 463-475.

15. Liu D, Diorio J, Tannenbaum B, Caldji C, Francis D, Freedman A et al. Maternal care, hippocampal glucocorticoid receptors, and hypothalamic-pituitary-adrenal responses to stress. Science 1997; 277: 1659-1662.

16. Francis D, Diorio J, Liu D, Meaney MJ. Nongenomic transmission across generations of maternal behavior and stress responses in the rat. Science 1999; 286: 1155-1158.

17. Weaver ICG, Cervoni N, Champagne FA, D'Alessio AC, Sharma S, Seckl JR et al. Epigenetic programming by maternal behavior. Nat Neurosci 2004; 7: 847-854.

18. McCormick JA, Lyons V, Jacobson MD, Noble J, Diorio J, Nyirenda M et al. $5^{\prime}$-heterogeneity of glucocorticoid receptor messenger RNA is tissue specific: differential regulation of variant transcripts by early-life events. Mol Endocrinol 2000; 14: 506-517.

19. Szyf M, Weaver ICG, Champagne FA, Diorio J, Meaney MJ. Maternal programming of steroid receptor expression and phenotype through DNA methylation in the rat. Fron Neuroendocrinol 2005; 26: 139-162.

20. Weaver ICG, D'Alessio AC, Brown SE, Hellstrom IC, Dymov S, Sharma $S$ et al. The transcription factor nerve growth factor-inducible protein a mediates epigenetic programming: altering epigenetic marks by immediate-early genes. J Neurosci 2007; 27: 1756-1768.

21. McGowan PO, Sasaki A, D’Alessio AC, Dymov S, Labonté B, Szyf M et al. Epigenetic regulation of the glucocorticoid receptor in human brain associates with childhood abuse. Nat Neurosci 2009; 12: 342-348.

22. Oberlander TF, Weinberg J, Papsdorf M, Grunau R, Misri S, Devlin AM. Prenatal exposure to maternal depression, neonatal methylation of human glucocorticoid receptor gene (NR3C1) and infant cortisol stress responses. Epigenetics 2008; 3: 97-106.

23. Stott DH. Follow-up study from birth of the effects of prenatal stresses. Dev Med Child Neurol 1973; 15: 770-787.

24. Ward AJ. Prenatal stress and childhood psychopathology. Child Psychiatry Hum Dev 1991; 22: $97-110$.

25. Roth M, Schauer M, Ruf M. Generationsübergreifende Folgen von Trauma und Posttraumatischer Belastungsstörung bei Flüchtlingen in Deutschland. Z Klin Psychol Psychother, submitted.

26. Hegarty K, Sheehan M, Schonfeld C. A multidimensional definition of partner abuse: development and preliminary validation of the composite abuse scale. J Fam Violence 1999; 14: 399-415.
27. Hegarty K, Fracgp, Bush R, Sheehan M. The composite abuse scale: further development and assessment of reliability and validity of a multidimensional partner abuse measure in clinical settings. Violence Vict 2005; 20: 529-547.

28. Taft A, Small R, Hegarty K, Lumley J, Watson L, Gold L. MOSAIC (MOthers' Advocates In the Community): protocol and sample description of a cluster randomised trial of mentor mother support to reduce intimate partner violence among pregnant or recent mothers. BMC Public Health 2009; 9: 159-159.

29. MacMillan $\mathrm{HL}$, Wathen $\mathrm{CN}$, Jamieson $\mathrm{E}$, Boyle MH, Shannon HS, Ford-Gilboe M et al. Screening for intimate partner violence in health care settings: a randomized trial. JAMA 2009; 302: 493-501.

30. Frommer M, McDonald LE, Millar DS, Collis CM, Watt F, Grigg GW et al. A genomic sequencing protocol that yields a positive display of 5-methylcytosine residues in individual. Proc Natl Acad Sci USA 1992; 89: 1827-1831.

31. Clark SJ, Harrison J, Paul CL, Frommer M. High-sensitivity mapping of methylated cytosines. Nucleic Acids Res 1994; 22: 2990-2997.

32. Boyle JS, Lew AM. An inexpensive alternative to glassmilk for DNA purification. Trends Genet 1995; 11: 8.

33. Sambrook J, Russell DW. Molecular Cloning. CSHL Press, 2001.

34. Moser D, Molitor A, Kumsta R, Tatschner T, Riederer P, Meyer J. The glucocorticoid receptor gene exon 1-F promoter is not methylated at the NGFI-A binding site in human hippocampus. World J Biol Psychiatry 2007; 8: 262-268.

35. Krug EG, Mercy JA, Dahlberg LL, Zwi AB, Lozano R. World report on violence and health. World Health Organization: Geneva, 2002.

36. Seckl JR, Meaney MJ. Glucocorticoid 'programming' and PTSD risk. Ann NY Acad Sci 2006; 1071: 351-378.

37. Kapoor A, Dunn E, Kostaki A, Andrews MH, Matthews SG. Fetal programming of hypothalamo-pituitary-adrenal function: prenatal stress and glucocorticoids. J Physiol 2006; 572: 31-44.

38. Dobbing J, Sands J. Comparative aspects of the brain growth spurt. Early Hum Dev 1979; 3: $79-83$.

39. Scarr S, McCartney K. How people make their own environments: a theory of genotype greater than environment effects. Child Dev 1983; 54: 424-435.

40. McGaugh JL. Memory and Emotion: the Making of lasting Memories. Columbia University Press: New York, USA, 2003.

Translational Psychiatry is an open-access journal published by Nature Publishing Group. This work is licensed under the Creative Commons Attribution-Noncommercial-No Derivative Works 3.0 Unported License. To view a copy of this license, visit http://creativecommons.org/licenses/by-nc-nd/3.0/

Supplementary Information accompanies the paper on the Translational Psychiatry website (http://www.nature.com/tp) 